\title{
Les journalists remplacent la verité par le mensonge, sous la pression des reseaux sociaux
}

\author{
Lecturer Horea Mihai BĂDĂU, PhD \\ Department of Journalisme \\ La Faculté de Journalisme et des Sciences de la Communication, \\ Université de Bucarest \\ E-mail: horeabadau@gmail.com
}

\begin{abstract}
Les typologies informationnelles qui ont du succès sur Facebook, fondées sur la production de l'émotion, peuvent-elles influencer le contenu généré par les sites d'infos? Peuvent-elles influencer de manière à ce que la recherche de la vérité, valeur principale de la culture professionnelle des journalistes, soit remplacée par la construction d'histoires pas vraies dont le but est de stimuler les instincts primaires? J'ai analysé les articles publiés sur les pages Facebook et sur les sites, à la rubrique "les infos les plus lues » des deux chaînes de télévision d'infos nationales: Realitatea TV et România TV, dans la période allant du 15 janvier au 5 fevrier 2016.

Conclusion: les valeurs journalistiques changent sous l'influence des réseaux sociaux. Aux côtés de la vérité, contenue par les genres d'opinions classiques, Société, Economie etc., le mensonge s'insinue, représenté par des nouveaux domaines: Bizarre/SF, Monde/Life et Erotisme. Un autre signe important du changement de la politique éditoriale, dans le sens du passage de la vérité au mensonge, c'est le grand nombre de faux titres et les infos auxquelles ils sont attribués. Les faux titres, en dehors du fait d'accompagner des articles des domaines Bizarre/SF, Mondanités, Erotisme, sont également utilisés comme préface à des articles appartenant à certains domaines traditionnels: Société, Politique, Economie), ce qui annonce la possible transition future de ces domaines de la vérité au mensonge.
\end{abstract}

Mots-cles: Journalisme; Verité; Mensonge; Reseaux Sociaux; Television. 


\section{Références bibliographiques}

1. Bădău, H.M. (2011), Tehnici de comunicare în Social Media, Iaşi, Ed. Polirom.

2. Bădău, H.M. (2015), Manual de Jurnalism Online, București, Ed. Tritonic.

3. Cardon D, Delaunay T.H., (2006), « La production de soi comme technique relationnelle. Un essai de typologie des blogs par leurs publics », Réseaux, 138, 15-71.

4. Coman, M (20017), Introducere in sistemul mass-media, Iași, Ed. Polirom.

5. Charaudeau, P., (1997), Le discours d'information mediatique. La construction du miroir social, Paris, Ed. Nathan.

6. Dacheux, E, (2007), Communiquer l'utopie, Paris, Ed. L'Harmattan.

7. Degenne A., Forsé M., (2004), Les réseaux sociaux. Une approche structurale en sociologie, Paris, Ed. Armand Colin.

8. Eco, U., (1997), Şase plimbări prin pădurea narativă, Constanta, Editura Pontica.

9. Gervais, J-F, (2007), Web 2.0 Les internautes au pouvoir, Paris, Ed. Dunod.

10. Rad, I., coord.(2016), Independența mass-media și libertatea de exprimare, Ed. Tritonic, Bucarest.

11. Rebillard, F., (2007), Le Web 2.0 en perspective, Paris, Ed Harmattan.

12. Tournier, M, (1967), Vendredi ou les Limbes du Pacifique, Paris, Ed. Gallimard.

13. Propp, Vladimir, (2015), Morphologie du conte, Paris, Ed. Seuil.

\section{Références bibliographiques en ligne}

14. www.ledauphine.com (vu le 01.05.2017)

15. www.imusiciandigital.com (vu le 01.05.2017)

16. http://nytmarketing.whsites.net (vu le 01.05.2017)

17. www.huffingtonpost.com (vu le 01.05.2017)

18. www.successfulblogging.com (vu le 01.05.2017)

19. www.blogdumoderateur.com (vu le 01.05.2017) 